\title{
Performance of $\mathrm{KCl}: \mathrm{Eu}^{2+}$ storage phosphor dosimeters for low dose measurements
}

\author{
H. Harold Lia ${ }^{a}$ Zhiyan Xiao, Rachael Hansel, Nels Knutson, and Deshan Yang \\ Department of Radiation Oncology, Washington University School of Medicine, 4921 Parkview \\ Place, Campus Box 8224, St. Louis, MO 63110
}

\begin{abstract}
Recent research has demonstrated that europium doped potassium chloride $\left(\mathrm{KCl}: \mathrm{Eu}^{2+}\right)$ storage phosphor material has the potential to become the physical foundation of a novel and reusable dosimetry system using either film-like devices or devices similar to thermoluminescent dosimeter (TLD) chips. The purposes of this work are to quantify the performance of $\mathrm{KCl}: \mathrm{Eu}^{2+}$ prototype dosimeters for low dose measurements and to demonstrate how it can be incorporated into clinical application for in vivo peripheral dose measurements. Pellet-style $\mathrm{KCl}: \mathrm{Eu}^{2+}$ dosimeters, $6 \mathrm{~mm}$ in diameter, and $1 \mathrm{~mm}$ thick, were fabricated in-house for this study. The dosimeters were read using a laboratory photostimulated luminescence detection system. $\mathrm{KCl}: \mathrm{Eu}^{2+}$ prototype storage phosphor dosimeter was capable of measuring a dose-to-water as low as $0.01 \mathrm{cGy}$ from a $6 \mathrm{MV}$ photon beam with a signal-to-noise ratio greater than 6 . A pre-readout thermal annealing procedure enabled the dosimeter to be read within an hour post irradiation. After receiving large accumulated doses $(\sim 10 \mathrm{kGy})$, the dosimeters retained linear response in the low dose region with only a 20 percent loss of sensitivity comparing to a fresh sample (zero Gy history). The energydependence encountered during low dose peripheral measurements could be accounted for via a single point outside-field calibration per each beam quality. With further development the $\mathrm{KCl}: \mathrm{Eu}^{2+-}$ based dosimeter could become a versatile and durable dosimetry tool with large dynamic range (sub-cGy to $100 \mathrm{~Gy}$ ).
\end{abstract}

\section{Keywords}

radiation therapy dosimetry; dosimeter; storage phosphor

\section{Introduction}

Modern radiation therapy dosimetry covers a wide range of dose, ranging from fraction of one cGy outside the primary radiation field, to the highest doses given in radiosurgery $(\sim 80$ Gy for trigeminal neuralgia radiosurgery). Many radiation therapy patients require in vivo peripheral dosimetry. For instance a physician may want to know the gonadal dose for young male patients, fetal dose for pregnant patients, or the pacemaker/defibrillator dose for patients with cardiac implants. Moreover, intensity modulated radiation therapy (IMRT) dosimetry requires a high spatial-resolution, reusable multidimensional dosimeter to characterize complex dose distributions with steep gradients. Reusability provides medical physicists a degree of confidence in dosimetric measurements through the acquisition of high-quality data, long-term repeated use, and performance monitoring. Recent research (Han et al., 2009; Zheng et al., 2010) has demonstrated that europium doped potassium 
chloride $\left(\mathrm{KCl}: \mathrm{Eu}^{2+}\right)$ storage phosphor material has the potential to become the physical foundation of a novel and reusable dosimetry system using either film-like devices or devices similar to thermoluminescent dosimeter chips. Developing such a versatile dosimetry system would provide the medical physics community with a unique resource for radiation therapy dosimetry.

$\mathrm{KCl}: \mathrm{Eu}^{2+}$ functions using photostimulated luminescence (PSL) mechanism (Rowlands, 2002; Thoms et al., 1991; Jursinic, 2007; Schembri and Heijmen, 2007). During x ray irradiation, electrons and holes are generated in numbers proportional to the $\mathrm{x}$ ray dose. These electrons and holes are stored in the material in charge-carrier storage centers such as $\mathrm{F}$-centers and $\mathrm{Eu}^{2+} / \mathrm{V}_{\mathrm{k}}$ centers. During the subsequent photostimulation, the electrons are energetically excited and released from the F-centers, and then recombine with the trapped holes. The energy released in this process excites $\mathrm{Eu}^{2+}$ to the $4 \mathrm{f}^{6} 5 \mathrm{~d}^{1}$ state with subsequent radiative decay to the $4 \mathrm{f}^{7}$ ground state, resulting in characteristic $\mathrm{Eu}^{2+}$ luminescence. Charge carriers remaining trapped after readout can be removed with a bright, broadband light and the dosimeter can be reused.

In this work, we will 1) evaluate the $\mathrm{KCl}: \mathrm{Eu}^{2+}$ prototype dosimeter's performance of measuring low dose and compare to commercially available $\mathrm{BaFBr}: \mathrm{Eu}^{2+}$ detectors, 2) demonstrate how the wait time between irradiation and readout can be reduced with a thermal treatment, 3) evaluate the dosimeter's energy-dependence and correction in peripheral regions, and 4) assess low dose measurement performance on dosimeters with high cumulated dose history. The data shown in this work will quantify the low dose detection resolution of the $\mathrm{KCl}: \mathrm{Eu}^{2+}$ prototype dosimeter and demonstrate how it can be incorporated into clinical application for in vivo peripheral dose measurements.

\section{Materials and methods}

\subsection{Dosimeters and irradiation}

The prototype $\mathrm{KCl}: \mathrm{Eu}^{2+}$ dosimeters used in this study were manufactured according to standard materials processes, and had approximately the same size and shape as thermoluminescent dosimeter chips. High purity $\mathrm{KCl}$ (99.995\%, Sigma-Aldrich) particles and reagent grade $\mathrm{EuCl}_{3}-6 \mathrm{H}_{2} \mathrm{O}$ particles were mixed at a $500 \mathrm{ppm} \mathrm{Eu}^{2+}$ concentration. Powders were pressed at $2200 \mathrm{lbs}$ force to $6 \mathrm{~mm}$ in diameter and $1 \mathrm{~mm}$ thick in a hydraulic press (Carver Inc., Wabash, IN) using an evaluable pressing die. The pellets were sintered at $710{ }^{\circ} \mathrm{C}$ for $3 \mathrm{~h}$ in air and allowed to cool naturally to $300{ }^{\circ} \mathrm{C}$ followed by a rapid cooling to room temperature. Dosimeters were wrapped in plastic wrap to mitigate the adverse effects of moisture on the material. Optical annealing with a $500 \mathrm{~W}$ tungsten-halogen lamp prior to irradiation removed background signal created through environmental irradiation, or residual signal from the previous measurement.

$\mathrm{KCl}: \mathrm{Eu}^{2+}$ dosimeters were irradiated using a multimodality linear accelerator (Trilogy, Varian Medical System, Palo Alto, CA, USA). To compare sensitivity, two commercial storage phosphor detectors, MD10 (Agfa Healthcare, Mortsel, Belgium) (Olch, 2005) and STIII (Fujifilm, Tokyo, Japan) (Dobbins et al., 1995), were also irradiated under the same conditions. The phantom consisted of solid water slabs (SW-457, Gammex RMI, Middleton, WI) with areas of $30 \times 30 \mathrm{~cm}^{2}$, stacked to yield $30 \mathrm{~cm}$ of thickness. Dosimeters were placed in a $5 \mathrm{~mm}$ thick slab with a linear array of holes machined $7.5 \mathrm{~mm}$ in diameter and $2 \mathrm{~mm}$ in depth. Three dosimeters were used for each dose point.

The lowest dose-to-water of $0.01 \mathrm{cGy}$ in this work was achieved underneath a fully closed multileaf collimator (MLC) at a source-to-surface distance (SSD) of $\sim 200 \mathrm{~cm}$. For peripheral dose measurements, a $30 \times 120 \times 20 \mathrm{~cm}^{3}$ phantom was constructed. The long 
dimension of the phantom placed on the treatment table corresponded to the superiorinferior axis of a patient lying on the treatment table. Peripheral dose distributions were measured along the long dimension of the phantom. Absolute dose-to-water was measured using an Accredited Dosimetry Calibration Laboratory (ADCL) calibrated $0.6 \mathrm{~cm}^{3}$ Farmertype ionization chamber (PTW N23333, Friedberg, Germany) connected to a calibrated electrometer (Keithley model 602, CNMC Co., Nashville, TN). Data were taken at distances up to $50 \mathrm{~cm}$ away from the primary radiation field edge.

For doses greater than $100 \mathrm{~Gy}$, prototype dosimeters were irradiated using the XRLM4 beamline at the Center for Advanced Mircrostructure and Devices synchrotron facility at Louisiana State University in Baton Rouge, LA. Monochromatic x ray beams of $30 \mathrm{keV}$ were selected by transporting the beam through a series of filters, including $175 \mu \mathrm{m} \mathrm{Be}$ window, $0.5 \mathrm{~mm}$ thick Ti filter, and $1 \mathrm{~mm}$ thick Al filter. Samples were mounted in a PMMA sample holder with holes of $1 \mathrm{~mm}$ depth and $7 \mathrm{~mm}$ diameter in an array of $2 \times 20$ holes. Spatially homogeneous irradiation of the dosimeters was achieved by mounting the sample holder to a motion stage controlled by a user-programmed LabVIEW (National Instruments Corporation, Austin, TX) and then vertically oscillating the irradiation target through the path of the beam. Doses were validated using EBT Gafchromic film (International Specialty Products, Wayne, NJ) (Brown et al., 2012).

\subsection{PSL readout system}

A $2 \mathrm{~mW}, 594 \mathrm{~nm} \mathrm{He}-\mathrm{Ne}$ laser (Melles Griot, Covina, CA) was used as readout light. The readout power was attenuated by neutral density (ND) filters as needed to allow for a large dynamic range. An electro-mechanic shutter was controlled through a General Purpose Interface Bus (GPIB) interface and used to switch the stimulation laser light on and off. The laser light was chopped with an optical chopper (Model SR540, Stanford Research System, Sunnyvale, CA) set to $150 \mathrm{~Hz}$ in order to provide a reference signal to a lock-in amplifier for phase sensitive detection. The stimulation light was focused by a series of lenses and directed to an integrating sphere with multiple ports (LabSphere, North Sutton, NH). The $\mathrm{KCl}: \mathrm{Eu}^{2+}$ dosimeter was mounted in a customized cap with an aperture of $7 \mathrm{~mm}$ on a port opposite the photomultiplier tube (PMT) which collected and amplified the PSL signals. The entire area of the dosimeter was uniformly illuminated by the stimulation light. The gain of the PMT was controlled through a direct current (DC) power supply (Model PS310, Stanford Research System) that supplied high voltage up to $1.25 \mathrm{kV}$. PSL signal was separated from readout light using a narrow bandpass filter FB420-10 (420 nm wavelength with $10 \mathrm{~nm}$ full-width-half-maximum, FWHM, Thorlabs, Newton, NJ) and detected by a PMT (R1924A, Hamamatsu, Bridgewater, NJ) connected to a lock-in amplifier (Model SR830, Stanford Research Systems). For Agfa and Fuji's detectors, a narrow bandpass filter FB390-10 was used (390 nm wavelength with $10 \mathrm{~nm}$ FWHM). The system was controlled through a GPIB interface. A typical measurement involved opening the shutter, waiting $1 \mathrm{~s}$ for the signal to stabilize, and then taking 64 consecutive readings. The mean of the 64 readings was recorded as a measurement.

Background signals were measured by placing unirradiated dosimeters on the integrating sphere. The background signals consist of a DC component and a random noise signal. The DC signal was largely due to the leakage of the ambient light (e.g., from a computer monitor) through the glass filters, luminescent centers generated by cosmic radiation and the bleaching lamp, as well as the PMT dark current. This DC signal can be removed by subtraction. The background noise, which is thermal and electronic in origin, ultimately defines the minimum detectable limit. 


\subsection{Readout schemes}

Two dosimetric readout schemes were investigated in this work. The first scheme requires waiting 17 hours post-irradiation before sample read-out. Previous results have shown that PSL intensity decreases with time after irradiation. The PSL fading curve typically consisting of a fast component immediately after irradiation and a much slower component afterward (Han et al., 2009). A long wait time, for example, $17 \mathrm{hrs}$ in this work eliminates the necessity of applying a large time-dependent correction. The exact moment of irradiation was recorded by a record-and-verify system (MOSAIQ, Elekta AB, Stockholm, Sweden); then a correction was applied to account for the difference in delay time between measurement dosimeters and calibration dosimeters.

Although reading the dosimeters on the following day works well in the clinical schedule, for in vivo dosimetry, reducing the wait time to few hours is desirable. Previous work has shown that a short thermal treatment can result in a fast stabilization of the PSL signal (Xiao et al., 2013). Therefore, the second readout scheme involved a pre-readout thermal annealing step. The dosimeters were irradiated by a linear accelerator while at room temperature, transferred to a laboratory oven (Binder, Bohemia, NY), and heated for 15 minutes at $70{ }^{\circ} \mathrm{C}$. The dosimeters were then read after a 15-min cooling-down to room temperature.

\section{Results}

\subsection{Performance of measuring low dose}

Table 1 shows measured doses underneath fully closed MLCs at different monitor unit (MU) settings using ionization chamber and $\mathrm{KCl}: \mathrm{Eu}^{2+}$. The output is estimated to be $0.0039 \mathrm{cGy}$ per MU at the dosimeter plane. The data demonstrates that $\mathrm{KCl}: \mathrm{Eu}^{2+}$ can accurately measure a doseto-water as low as $0.01 \mathrm{cGy}$. For current PSL readout optics, the background was about $1 \mu \mathrm{V}$ with noise of about $0.3 \mu \mathrm{V}$, the latter being the main limiting factor for the $\mathrm{KCl}: \mathrm{Eu}^{2+}$ dosimeter's dose resolution and uncertainty. A $0.01 \mathrm{cGy}$ dose-to-water yielded a net signal level at about $2 \mu \mathrm{V}$ after background subtraction. Other type A uncertainties ( $\mathrm{Li}$ et al., 2007) associated with $\mathrm{KCl}: \mathrm{Eu}^{2+}$ prototypes include positioning uncertainty due to manual placement of dosimeters in the readout system, reproducibility of the reader, and performance variation among dosimeters. These uncertainties can certainly be reduced by improved instrumentation and materials engineering. Type B uncertainty is related to ionization chamber calibration, which is of the order of 1-2\% (Almond et al., 1999).

Agfa's MD10, Fuji's STIII and KCl:Eu ${ }^{2+}$ prototypes were within $10 \%$ in sensitivity after irradiation to the same dose-to-water of $5 \mathrm{cGy}$. Note that all three were read on the second day after irradiation. Same results were obtained after another irradiation to a dose-to-water of 9 cGy.

\subsection{Wait time between irradiation and dosimeter readout}

Figure 1 shows the dose calibration curves for the two different readout schemes over the dose range of $1 \mathrm{cGy}$ to $10 \mathrm{cGy}$. The two curves are linear and overlap with each other. The first curve was acquired $17 \mathrm{hrs}$ post-irradiation when the fading-rate was approximately $0.5 \%$ per hr. Since the fading-rate is quite low on the second day and it typically takes less than 1 minute to read a dosimeter, even without any correction, the introduced error would be less than half a percent. The second curve was acquired within $1 \mathrm{hr}$ post-irradiation after a 15 min pre-readout thermal annealing at $70^{\circ} \mathrm{C}$. The two readout schemes yielded the same result, providing the user with the flexibility of reading dosimeters according to the work schedule. 


\subsection{Energy-correction in the peripheral region}

Table 2 shows peripheral dose measurements using $\mathrm{KCl}: \mathrm{Eu}^{2+}$ vs. ionization chamber for a 6 $\mathrm{MV}$ beam. Clearly, $\mathrm{KCl}: \mathrm{Eu}^{2+}$ reported much higher dose than ionization chamber. However, if the dosimeter is calibrated outside of field, here $10 \mathrm{~cm}$ from the field edge as shown in column 4, the doses reported by $\mathrm{KCl}: \mathrm{Eu}^{2+}$ were much closer to those reported by ionization chamber. This suggests that a single outside-field calibration is sufficient for correcting energy-dependence in the peripheral region. The outside-field calibration is obtained by determining a factor that relates the ion chamber measurement to the $\mathrm{KCl}: \mathrm{Eu}^{2+}$ measurement. This factor is determined by dividing the $\mathrm{KCl}: \mathrm{Eu}^{2+}$-measured dose at $10 \mathrm{~cm}$ distance from the field edge by the ion chamber-measured dose at the same location. For example, in Table 2 the outside-field calibration factor is 1.56 . All subsequent $\mathrm{KCl}: \mathrm{Eu}^{2+}$ measured doses at various distances are then divided by this factor to obtain the corrected doses. Comparable magnitude of error was reported for the peripheral dose measurements using a MOSFET dosimeter, highlighting the importance of appropriate calibration of a dosimeter with modest atomic number (Z) (Butson et al., 2005).

Table 3 shows peripheral dose measurements using $\mathrm{KCl}: \mathrm{Eu}^{2+} \mathrm{vs}$. ionization chamber for a $10 \mathrm{MV}$ beam. Although the magnitude of over-response is smaller than for $6 \mathrm{MV}$ (45\% vs. $56 \%$ ), an over-response is still evident. Similarly, a single outside-field calibration (factor = 1.45) reduced the error to $0.05 \mathrm{cGy}$ or less.

Tables 4 and 5 show peripheral dose measurements using $\mathrm{KCl}: \mathrm{Eu}^{2+}$ vs. ionization chamber for a $18 \mathrm{MV}$ beam at two different bolus thickness, $1 \mathrm{~cm}$ and $3.5 \mathrm{~cm}$, respectively. If placed underneath a $1 \mathrm{~cm}$ bolus, dosimeter's over-response is minimal (Table 4), indicating the inconsequential photon spectrum variation between in-field and outside-field for an $18 \mathrm{MV}$ beam with slim bolus thickness. By contrast, if placed underneath a bulky $3.5 \mathrm{~cm}$ bolus, the dosimeter over-responded by $29 \%$ due to significant increased phantom scattering. Again, an outside-field calibration (factor $=1.29$ ) is sufficient to reduce the error to $0.11 \mathrm{cGy}$ or less.

The reason to study bolus-thickness effect is due to the large change in absorbed dose between skin and dmax. As shown in Table 6, peripheral dose changes with depth especially in the buildup region. For example, for an $18 \mathrm{MV}$ beam, dose at $1 \mathrm{~cm}$ depth is almost three times larger than that at deeper depths. Thus, the dose measured with a $1 \mathrm{~cm}$ thick bolus represented the maximum dose to a patient while the dose measured with a $3.5 \mathrm{~cm}$-thick bolus was more representative of the dose at deeper depth in the patient. Therefore, depending on clinical interest, different bolus thickness may be used, which in turn affects $\mathrm{KCl}: \mathrm{Eu}^{2+}$ dosimeter's response.

\subsection{Low-dose performance retention at high cumulated dose}

Figure 2 shows the dose response curves at cumulated doses of $5 \mathrm{kGy}$ and $10 \mathrm{kGy}$. Despite large dose history, dosimeters retained linear response in the low dose region with only a 20 percent loss of sensitivity comparing to a fresh sample (zero Gy history).

\section{Discussion and conclusion}

$\mathrm{KCl}$ has a higher effective $\mathrm{Z}$ than water, 18 vs. 7. However, Monte-Carlo simulations demonstrate that reducing the thickness of the $\mathrm{KCl}: \mathrm{Eu}^{2+}$ dosimeter to a few microns would convert a Burlin-cavity dosimeter to a Bragg-Gray dosimeter (Zheng et al., 2010). As a result, $\mathrm{KCl}: \mathrm{Eu}^{2+}$ with a thin geometry on the order of a few microns can provide almost noenergy dependence for mega-voltage therapeutic beam dosimetry. Future development would involve the creation of a large-area $\mathrm{KCl}: \mathrm{Eu}^{2+}$-based storage phosphor panel using modern thin film techniques, for example, tape casting (Li et al., 2002; Mistler and 
Twiname, 2001) or physical vapor deposition (Hell et al., 2008; Schmitt et al., 2002; Leblans et al., 2000). However, decreasing thickness results in a reduction in sensitivity (Fasbender et al., 2003). Thus, if $\mathrm{KCl}: \mathrm{Eu}^{2+}$ at such a thin-geometry is still sufficiently sensitive remains to be experimentally demonstrated.

The data from this work shows that $\mathrm{KCl}: \mathrm{Eu}^{2+}$ material exhibits excellent ionizing-radiation storage capability that is comparable to commercially available $\mathrm{BaFBr}: \mathrm{Eu}^{2+}$-based storage phosphor detectors but has a much smaller $\mathrm{Z}$. $\mathrm{KCl}: \mathrm{Eu}^{2+}$ dosimeters are capable of resolving a dose-to-water of $0.01 \mathrm{cGy}$ from a megavoltage beam. Therefore, one can expect that a thin-dosimeter on the order a few microns would be able to detect a dose on the order of one cGy vs. daily therapeutic dose of 200 cGy. Moreover, current laboratory data demonstrates that $\mathrm{KCl}: \mathrm{Eu}^{2+}$ exhibits a linear response from $1 \mathrm{~Gy}$ to $100 \mathrm{~Gy}$. More importantly, $\mathrm{KCl}: \mathrm{Eu}^{2+}$ based system demonstrates strong radiation hardness. These data collectively support further development of such a dosimetry system.

For low peripheral dose measurements, chip-style, thick $\mathrm{KCl}: \mathrm{Eu}^{2+}$ dosimeters are more useful. However, its high atomic number makes it sensitive to variation in the photon spectrum. There is significant amount of low-energy scattered photons in the peripheral region, causing $\mathrm{KCl}: \mathrm{Eu}^{2+}$ dosimeter's strong over-response. It is recommended that the dosimeter be calibrated outside the primary radiation field using the dose-to-water value measured by an ionization chamber since in-field photon energy differs substantially from that of the peripheral region. Our data shows that a single outside-field calibration is sufficient to reduce dose measurement error to $0.1 \mathrm{cGy}$ or less. This is consistent with Kry et al s simulation results (Kry et al., 2006; Kry et al., 2007). They reported that for 6 MV beams, the average photon energy on central axis was $1.50 \mathrm{MeV}$; at distances between 10 and $50 \mathrm{~cm}$ from the central axis the average photon energy was nearly constant, increasing slightly from $0.359 \mathrm{MeV}$ at $10 \mathrm{~cm}$ to $0.392 \mathrm{MeV}$ at $30 \mathrm{~cm}$ and $0.41 \mathrm{MeV}$ at $50 \mathrm{~cm}$. For 18 MV beams, at $10 \mathrm{~cm}$ from the central axis, the average photon energy was $0.74 \mathrm{MeV}$, while at both 30 and $50 \mathrm{~cm}$ from the central axis, the average energy was $0.57 \mathrm{MeV}$. Taking into account $\mathrm{KCl}: \mathrm{Eu}^{2+}$ 's mass attenuation coefficient vs. photon-energy data (cf. Figure 7 of Ref. (Zheng et al., 2010)), the photon spectrum doesn't change enough to significantly affect the response of the dosimeter. To further improve measurement accuracy, systematic energydependence measurements combined with Monte-Carlo simulations should be conducted. For example, Scarboro et al. reported that outside the treatment field a correction factor ranging from 0.88 to 0.99 needs to be applied for LiF-TLDs depending on the specific irradiation conditions (Scarboro et al., 2011). Also, cross-calibration with an ionization chamber might not always be possible for the desired measurement positions, for which a Monte-Carlo model is helpful.

In conclusion, $\mathrm{KCl}: \mathrm{Eu}^{2+}$ prototype storage phosphor dosimeter is capable of measuring a dose-to-water as low as $0.01 \mathrm{cGy}$ for a megavoltage photon beam. A pre-readout thermal annealing procedure at $70^{\circ} \mathrm{C}$ for 15 minutes is implemented which enables the dosimeter available for readout within an hour post irradiation, compared to $17 \mathrm{hr}$ wait time without intervention. Due to dosimeter's larger atomic number relative to that of water, an out-offield calibration for each beam energy is required to minimize the dosimeter's overresponse. The dosimeter retains low-dose measuring capability after a cumulated dose of 10 $\mathrm{kGy}$. With further development the $\mathrm{KCl}: \mathrm{Eu}^{2+}$ - based dosimeter could become a versatile and durable tool with a wide range of applications in the modern radiation oncology clinic.

\section{Acknowledgments}

This work was supported in part by NIH Grant No. R01CA148853. 


\section{REFERENCES}

Almond PR, Biggs PJ, Coursey BM, Hanson WF, Huq MS, Nath R, Rogers DW. AAPM's TG-51 protocol for clinical reference dosimetry of high-energy photon and electron beams. Med Phys. 1999; 26:1847-1870. [PubMed: 10505874]

Brown TA, Hogstrom KR, Alvarez D, Matthews KL 2nd, Ham K, Dugas JP. Dose-response curve of EBT EBT2, and EBT3 radiochromic films to synchrotron-produced monochromatic xray beams. Med Phys. 2012; 39:7412-7417. [PubMed: 23231291]

Butson MJ, Cheung T, Yu PK. Peripheral dose measurement with a MOSFET detector. Appl Radiat Isot. 2005; 62:631-634. [PubMed: 15701420]

Dobbins JT 3rd, Ergun DL, Rutz L, Hinshaw DA, Blume H, Clark DC. DQE(f) of four generations of computed radiography acquisition devices. Med Phys. 1995; 22:1581-1593. [PubMed: 8551982]

Fasbender R, Li H, Winnacker A. Monte Carlo modeling of storage phosphor plate readouts. Nucl. Instr. and Meth. in Phys. Res. A. 2003; 512:610-618.

Han Z, Driewer JP, Zheng Y, Low DA, Li H. Quantitative megavoltage radiation therapy dosimetry using the storage phosphor KCl: Eu ${ }^{2+}$. Med Phys. 2009; 36:3748-3757. [PubMed: 19746808]

Hell, E.; Fuchs, M.; Mattern, D.; Schmitt, B.; Leblans, P. Binderless storage phosphor screen with needle shaped crystals. US Patent. 7,422,765 B2. 2008.

Jursinic PA. Characterization of optically stimulated luminescent dosimeters, OSLDs, for clinical dosimetric measurements. Med Phys. 2007; 34:4594-4604. [PubMed: 18196786]

Kry SF, Titt U, Followill D, Ponisch F, Vassiliev ON, White RA, Stovall M, Salehpour M. A Monte Carlo model for out-of-field dose calculation from high-energy photon therapy. Med Phys. 2007; 34:3489-99. [PubMed: 17926952]

Kry SF, Titt U, Ponisch F, Followill D, Vassiliev ON, White RA, Mohan R, Salehpour M. A Monte Carlo model for calculating out-of-field dose from a varian 6 MV beam. Med Phys. 2006; 33:4405-4413. [PubMed: 17153419]

Leblans P, Struye L, Willems P. A new needle-crystalline computed radiography detector. J Digit Imaging. 2000; 13:117-120. [PubMed: 10847378]

Li H, Epelbaum E, Batentschuk M, Winnacker A. Tape casting of storage phosphor BaFBr:Eu ${ }^{2+}$ for X-ray imaging. Mater. Sci. Eng. B. 2002; 96:313-319.

Li H, Gonzalez AL, Ji H, Duggan DM. Dose response of BaFBrI:Eu ${ }^{2+}$ storage phosphor plates exposed to megavoltage photon beams. Med. Phys. 2007; 34:103-111. [PubMed: 17278495]

Mistler RE, Twiname ER. Tape Casting: Theory and Practice. John Wiley \& Sons. 2001

Olch AJ. Evaluation of a computed radiography system for megavoltage photon beam dosimetry. Med Phys. 2005; 32:2987-2999. [PubMed: 16266113]

Rowlands JA. The physics of computed radiography. Phys Med Biol. 2002; 47:R123-R166. [PubMed: 12502037]

Scarboro SB, Followill DS, Howell RM, Kry SF. Variations in photon energy spectra of a 6 MV beam and their impact on TLD response. Med Phys. 2011; 38:2619-2628. [PubMed: 21776799]

Schembri V, Heijmen BJ. Optically stimulated luminescence (OSL) of carbon-doped aluminum oxide $\left(\mathrm{Al}_{2} \mathrm{O}_{3}: \mathrm{C}\right)$ for film dosimetry in radiotherapy. Med Phys. 2007; 34:2113-2118. [PubMed: 17654914]

Schmitt B, Fuchs M, Hell E, Knüpfer W, Hackenschmied P, Winnacker A. Structured alkali halides for medical applications. Nucl. Instr. Meth. Phys. Res. B. 2002; 191:800-804.

Thoms M, von Seggern H, Winnacker A. Spatial correlation and photostimulability of defect centers in the x-ray-storage phosphor BaFBr:Eu ${ }^{2+}$. Phys. Rev. B. 2002; 44:9240-9247.

Xiao Z, Hansel R, Chen H, Du D, Yang D, Li H. Temporal signal stability of KCl:Eu ${ }^{2+}$ storage phosphor dosimeters. Med Phys. 2013; 40021721.

Zheng Y, Han Z, Driewer JP, Low DA, Li H. Theoretical and empirical investigations of $\mathrm{KCl}: \mathrm{Eu}^{2+}$ for nearly water-equivalent radiotherapy dosimetry. Med Phys. 2010; 37:146-153. [PubMed: 20175476] 


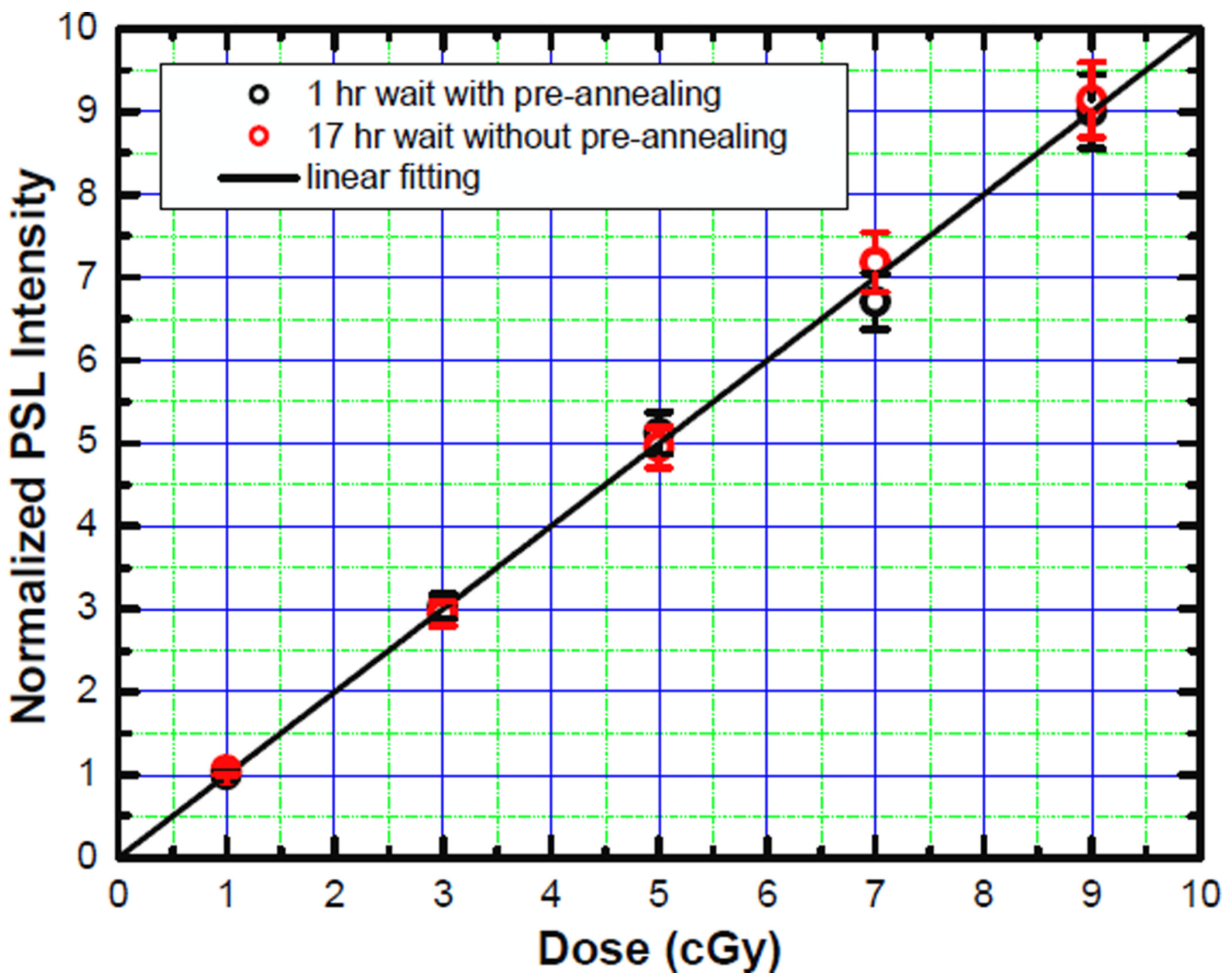

Figure 1.

PSL intensity vs. dose-to-water. The first data set was acquired within $1 \mathrm{hr}$ post irradiation with pre-readout thermal annealing, while the second data set was acquired with a $17 \mathrm{hr}$ wait but without pre-readout thermal annealing. $\mathrm{R}^{2}$ of the linear fitting: 0.9984 for $1 \mathrm{~h}$ wait with pre-annealing and 0.992 for $17 \mathrm{~h}$ wait without pre-annealing. 


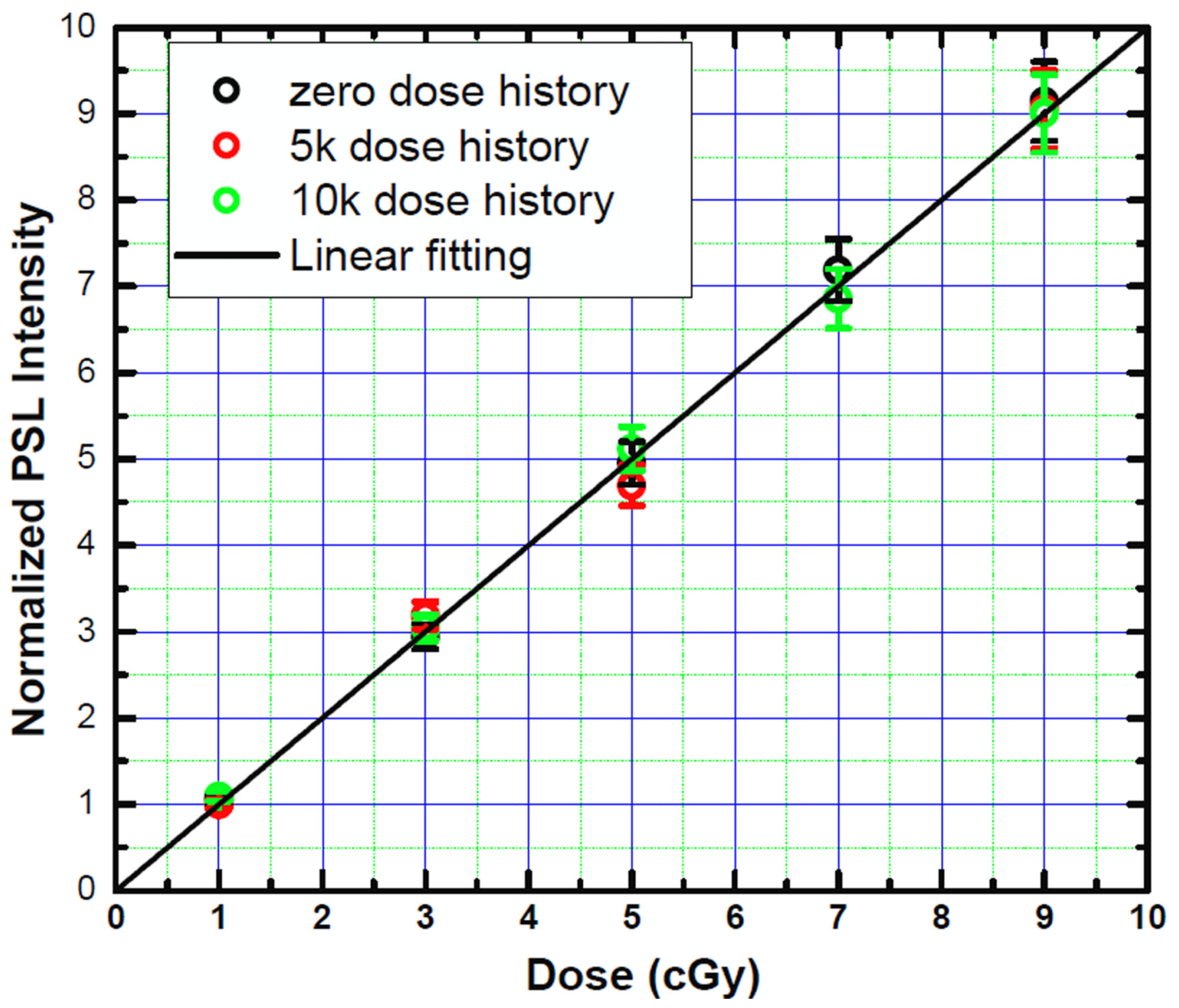

Figure 2.

PSL vs. dose-to-water as a function of dosimeter's prior dose history. Dosimeter retained low-dose measuring performance with up to $20 \%$ loss in sensitivity. $\mathrm{R}^{2}$ of the linear fitting: 0.992 for zerodose history, 0.9963 for $5 \mathrm{k}$ Gy history and 0.9981 for 10k Gy history. 


\section{Table 1}

$\mathrm{SSD}=200 \mathrm{~cm}$, MLC fully closed, dose rate: $100 \mathrm{MU} / \mathrm{min}$, field size: $14 \times 14 \mathrm{~cm}^{2}$. Ionization chamber value has an approximately 1-2\% type B uncertainty and the value of the coverage factor $\mathrm{k}$ is $1 . \mathrm{KCl}: \mathrm{Eu}^{2+}$ values represent the mean \pm standard deviation of repeated measurements using multiple dosimeters.

\begin{tabular}{cccc}
\hline MU setting & Ionization chamber $(\mathbf{c G y})$ & $\mathbf{K C l}_{\mathbf{E}} \mathbf{E u}^{2+}(\mathbf{c G y})$ & Error $(\mathbf{c G y})$ \\
\hline $3 \mathrm{MU}$ & 0.012 & $0.013 \pm 0.002$ & 0.001 \\
$9 \mathrm{MU}$ & 0.035 & $0.030 \pm 0.003$ & 0.005 \\
$27 \mathrm{MU}$ & 0.110 & $0.122 \pm 0.013$ & 0.012 \\
$36 \mathrm{MU}$ & 0.142 & $0.138 \pm 0.012$ & 0.004 \\
\hline
\end{tabular}


Table 2

Out-of-field measurements for a $15 \times 15 \mathrm{~cm}^{2} 6 \mathrm{MV}$ beam in a phantom as a function of distance from the radiation field edge. Bolus thickness: $1.5 \mathrm{~cm}$, MU: 300 MUs, SSD: $100 \mathrm{~cm}$.

\begin{tabular}{ccccc}
\hline $\begin{array}{c}\text { Distance from } \\
\text { field edge }(\mathbf{c m})\end{array}$ & $\begin{array}{c}\text { Ionization } \\
\text { chamber (cGy) }\end{array}$ & $\begin{array}{c}\text { KCl:Eu }{ }^{2+}(\text { in-field } \\
\text { calibration) }(\mathbf{c G y})\end{array}$ & $\begin{array}{c}\text { KCl:Eu } \\
\text { calibration) }(\mathbf{c G y})\end{array}$ & $\begin{array}{c}\text { Error } \\
(\mathbf{c G y})\end{array}$ \\
\hline 10 & 5.53 & $8.63 \pm 0.43$ & $5.53 \pm 0.27$ & N/A \\
20 & 1.77 & $2.74 \pm 0.22$ & $1.76 \pm 0.14$ & 0.01 \\
30 & 0.66 & $1.15 \pm 0.11$ & $0.74 \pm 0.07$ & 0.08 \\
40 & 0.37 & $0.57 \pm 0.07$ & $0.36 \pm 0.05$ & 0.01 \\
48.5 & 0.18 & $0.34 \pm 0.02$ & $0.22 \pm 0.01$ & 0.04 \\
\hline
\end{tabular}


Table 3

Out-of-field measurements for a $15 \times 15 \mathrm{~cm}^{2} 10 \mathrm{MV}$ beam in a phantom as a function of distance from the radiation field edge. Bolus thickness: $2 \mathrm{~cm}$, MU: 300 MUs, SSD: $100 \mathrm{~cm}$.

\begin{tabular}{ccccc}
\hline $\begin{array}{c}\text { Distance from } \\
\text { field edge }(\mathbf{c m})\end{array}$ & $\begin{array}{c}\text { Ionization } \\
\text { chamber (cGy) }\end{array}$ & $\begin{array}{c}\mathrm{KCl}: \mathrm{Eu}^{2+} \text { (in-field } \\
\text { calibration) }(\mathbf{c G y})\end{array}$ & $\begin{array}{c}\mathrm{KCl:Eu}{ }^{2+} \text { (outside-field } \\
\text { calibration) }(\mathbf{c G y})\end{array}$ & $\begin{array}{c}\text { Error } \\
\text { (cGy) }\end{array}$ \\
\hline 10 & 4.78 & $6.92 \pm 0.28$ & $4.78 \pm 0.19$ & N/A \\
20 & 1.60 & $2.25 \pm 0.17$ & $1.56 \pm 0.12$ & 0.04 \\
30 & 0.67 & $1.03 \pm 0.09$ & $0.71 \pm 0.06$ & 0.04 \\
40 & 0.39 & $0.56 \pm 0.02$ & $0.38 \pm 0.01$ & 0.01 \\
48.5 & 0.20 & $0.36 \pm 0.03$ & $0.25 \pm 0.02$ & 0.05 \\
\hline
\end{tabular}




\section{Table 4}

Out-of-field measurements for a $15 \times 15 \mathrm{~cm}^{2} 18 \mathrm{MV}$ beam in a phantom as a function of distance from the radiation field edge. Bolus thickness: $1 \mathrm{~cm}$, MU: 300 MUs, SSD: $100 \mathrm{~cm}$.

\begin{tabular}{cccc}
\hline $\begin{array}{c}\text { Distance from } \\
\text { field edge }(\mathbf{c m})\end{array}$ & $\begin{array}{c}\text { Ionization } \\
\text { chamber (cGy) }\end{array}$ & $\begin{array}{c}\text { KCl:Eu }{ }^{2+} \text { (in-field } \\
\text { calibration) }(\mathbf{c G y})\end{array}$ & Error $(\mathbf{c G y})$ \\
\hline 10 & 12.96 & $12.25 \pm 0.06$ & 0.71 \\
20 & 4.43 & $4.25 \pm 0.03$ & 0.18 \\
30 & 1.41 & $1.50 \pm 0.02$ & 0.09 \\
40 & 0.59 & $0.75 \pm 0.01$ & 0.16 \\
48.5 & 0.29 & $0.41 \pm 0.05$ & 0.12 \\
\hline
\end{tabular}


Table 5

Out-of-field measurements for a $15 \times 15 \mathrm{~cm}^{2} 18 \mathrm{MV}$ beam in a phantom as a function of distance from the radiation field edge. Bolus thickness: $3.5 \mathrm{~cm}$, MU: 300 MUs, SSD: $100 \mathrm{~cm}$.

\begin{tabular}{ccccc}
\hline $\begin{array}{c}\text { Distance from } \\
\text { field edge }(\mathbf{c m})\end{array}$ & $\begin{array}{c}\text { Ionization } \\
\text { chamber (cGy) }\end{array}$ & $\begin{array}{c}\text { KCl:Eu }{ }^{2+}(\text { in-field } \\
\text { calibration) }(\mathbf{c G y})\end{array}$ & $\begin{array}{c}\text { KCl:Eu}{ }^{2+}(\text { outside-field } \\
\text { calibration) }(\mathbf{c G y})\end{array}$ & $\begin{array}{c}\text { Error } \\
(\mathbf{c G y})\end{array}$ \\
\hline 10 & 4.80 & $6.21 \pm 0.27$ & $4.80 \pm 0.21$ & N/A \\
20 & 1.61 & $2.22 \pm 0.16$ & $1.72 \pm 0.12$ & 0.11 \\
30 & 0.67 & $0.97 \pm 0.06$ & $0.75 \pm 0.05$ & 0.08 \\
40 & 0.42 & $0.69 \pm 0.03$ & $0.53 \pm 0.02$ & 0.11 \\
48.5 & 0.25 & $0.39 \pm 0.01$ & $0.30 \pm 0.01$ & 0.05 \\
\hline
\end{tabular}




\section{Table 6}

Out-of-field measurements using ionization chamber for $15 \times 15 \mathrm{~cm}^{2}$ beams in a phantom as a function of depth from the phantom surface. MU: 300 MUs, SSD: $100 \mathrm{~cm}$, distance-to-field edge: $10 \mathrm{~cm}$

\begin{tabular}{cccc}
\hline Depth $(\mathbf{c m})$ & 6X (cGy) & 10X (cGy) & 18X (cGy) \\
\hline 1.0 & 5.47 & 8.13 & 12.94 \\
1.5 & 3.98 & -- & -- \\
2.0 & 3.98 & 4.81 & 7.74 \\
3.0 & -- & -- & 5.53 \\
3.5 & -- & -- & 4.80 \\
8.0 & 4.59 & 4.31 & 3.81 \\
15.0 & 4.70 & -- & 3.87 \\
\hline
\end{tabular}

Phys Med Biol. Author manuscript; available in PMC 2014 June 21. 